\title{
Exploring the role of CT densitometry: a randomised study of augmentation therapy in $\alpha_{1}$-antitrypsin deficiency
}

\author{
A. Dirksen*, E. Piitulainen\#, D.G. Parr`, C. Deng ${ }^{+}$, M. Wencker ${ }^{\S}$, \\ S.B. Shaker* and R.A. Stockley
}

ABSTRACT: Assessment of emphysema-modifying therapy is difficult, but newer outcome measures offer advantages over traditional methods. The EXAcerbations and Computed Tomography scan as Lung End-points (EXACTLE) trial explored the use of computed tomography (CT) densitometry and exacerbations for the assessment of the therapeutic effect of augmentation therapy in subjects with $\alpha_{1}$-antitrypsin $\left(\alpha_{1}\right.$-AT) deficiency.

In total, 77 subjects (protease inhibitor type Z) were randomised to weekly infusions of $60 \mathrm{mg} \cdot \mathrm{kg}^{-1}$ human $\alpha_{1}$-AT (Prolastin ${ }$ ) or placebo for 2-2.5 yrs. The primary end-point was change in CT lung density, and an exploratory approach was adopted to identify optimal methodology, including two methods of adjustment for lung volume variability and two statistical approaches. Other end-points were exacerbations, health status and physiological indices.

CT was more sensitive than other measures of emphysema progression, and the changes in CT and forced expiratory volume in $1 \mathrm{~s}$ were correlated. All methods of densitometric analysis concordantly showed a trend suggestive of treatment benefit ( $p$-values for Prolastin ${ }$ versus placebo ranged 0.049-0.084). Exacerbation frequency was unaltered by treatment, but a reduction in exacerbation severity was observed.

In patients with $\alpha_{1}$-AT deficiency, CT is a more sensitive outcome measure of emphysemamodifying therapy than physiology and health status, and demonstrates a trend of treatment benefit from $\alpha_{1}$-AT augmentation.

KEYWORDS: $\alpha_{1}$-Antitrypsin deficiency, computed tomography, emphysema, exacerbations, lung densitometry, lung function

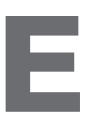
mphysema is believed to occur in individuals with $\alpha_{1}$-antitrypsin $\left(\alpha_{1}\right.$-AT) deficiency, primarily because of an inability to control neutrophil elastase released in the lung, thereby causing excessive tissue proteolysis and progressive alveolar destruction [1]. The hypothesis that emphysema progression could be reduced by redressing this imbalance has led to the development of plasma purification processes for $\alpha_{1}$-AT augmentation therapy for use in $\alpha_{1}$-AT deficiency in a number of countries.

A randomised placebo-controlled trial of $\alpha_{1}$-AT augmentation therapy with traditional physiological measures used as the primary end-point has not been undertaken because such a study is considered impractical [2, 3]; this therapy is therefore currently justified by clinical logic, proven biochemical efficacy [4] and data from well-controlled observational studies that are suggestive of treatment efficacy [5-8]. Forced expiratory volume in $1 \mathrm{~s}$ (FEV1) has been regarded as the "gold standard" outcome measure as a surrogate marker for emphysema progression; however, in order to detect a difference in FEV1 decline of $15 \mathrm{~mL} \cdot \mathrm{yr}^{-1}$, it has been estimated that 494 subjects with a baseline FEV1 of $35-79 \%$ predicted would be required per treatment arm over 3 yrs [2]. The change in computed tomography (CT) lung density has been shown to be a more sensitive and specific alternative measure than FEV1 [9], and a randomised controlled efficacy study with change in lung density as a secondary end-point has been undertaken [10]. Although this latter study was not suitably powered to demonstrate a treatment
AFFILIATIONS

*Gentofte Hospital, Copenhagen University, Hellerup, Denmark. \#University Hospital, Malmö, Sweden.

-University Hospitals of Coventry and Warwickshire and

fUniversity Hospitals of Birmingham UK

${ }^{+}$Talecris Biotherapeutics Inc Research Triangle Park, NC, USA ${ }^{\S}$ Talecris Biotherapeutics $\mathrm{GmbH}$, Frankfurt, Germany.

CORRESPONDENCE

A. Dirksen, Gentofte Hospital, Copenhagen University, DK-2900 Hellerup, Denmark. Fax: 45 39777693

E-mail: adi@dadlnet.dk

Received

October 222008

Accepted after revision:

January 122009

\section{SUPPORT STATEMENT}

This study was sponsored by Talecris Biotherapeutics, Inc. (Research

Triangle Park, NC, USA). S.B. Shaker has received a research grant from the Alpha-1 Foundation (Miami, FL, USA).

\section{CLINICAL TRIAL}

Registered in ClinicalTrials.gov as "Alpha-1-Antitrypsin (AAT) to Treat Emphysema in AAT-Deficient Patients"; Identifier: NCT00263887 The trial was conducted between November 2003 and January 2007

\section{STATEMENT OF INTEREST}

Statements of interest for A. Dirksen, C. Deng, M. Wencker and R.A. Stockley, and the study itself, can be found at www.erj.ersjournal.com/ misc/statements.dtl 
effect, it did indicate that if CT scan was the primary outcome measure, a study of intervention in emphysema could be conducted over a shorter timescale in fewer patients than are needed for a physiological outcome.

CT scanning has been validated as an in vivo measure of emphysema by studies that have shown good correlation with pathology [11, 12], exercise capacity [13], health status [13] and lung function $[14,15]$. Furthermore, while CT densitometry is a more sensitive measure of emphysema progression and a better predictor of mortality than lung function [16], CT lung density decline also relates to progressive reduction in FEV1 [14]. Although these studies have led to the acceptance of this relatively novel technique, subsequent technological advances and a continued lack of consensus on methodology make further exploratory studies necessary.

The aim of the current study (the EXAcerbations and CT scan as Lung End-points (EXACTLE) trial) was to assess various surrogate outcome measures for the progression of emphysema, with focus on CT lung densitometry. In order to make the comparison as realistic as possible, we decided to carry it out as part of a randomised, double-blind, placebo-controlled clinical trial of the effects of $\alpha_{1}$-AT augmentation therapy in patients with severe $\alpha_{1}$-AT deficiency. The primary outcome was change in whole lung density between the two treatment groups measured by CT. Additionally, different methods for lung volume correction were explored. Other outcomes included lung function, health status and exacerbations of chronic obstructive pulmonary disease (COPD).

\section{MATERIALS AND METHODS \\ Patients}

Patients with severe congenital $\alpha_{1}$-AT deficiency (all with $\alpha_{1}$ AT serum concentration $<11 \mu \mathrm{M}$ ) were recruited from $\alpha_{1}$-AT registries in Denmark, the UK and Sweden. Inclusion and exclusion criteria are reported in the online supplementary material. The study was approved by the Ethics Committee of The Capital Region of Denmark (Hillerød, Denmark), the National Health Service West Midlands South Birmingham Research Ethics Committee (Birmingham, UK) and the Regional Ethics Committee of Lund (Lund, Sweden). It was conducted in accordance with the Declaration of Helsinki and Good Clinical Practice guidelines, and all patients gave written informed consent.

\section{Study design}

This was a randomised, double-blind, placebo-controlled, parallel-group study conducted at three European centres (Copenhagen (Denmark), Birmingham (UK) and Malmö (Sweden)). Eligible patients were randomly assigned to weekly infusions of either $\alpha_{1}$-AT (Prolastin ${ }_{\circledR}$; Talecris Biotherapeutics, Inc., Research Triangle Park, NC, USA; $60 \mathrm{mg} \cdot \mathrm{kg}^{-1}$ body weight) or placebo ( $2 \%$ albumin), in permuted blocks of four with stratification according to country, for 24 months, with an optional extension to 30 months in subjects who agreed to continue in the study. After randomisation, patients were seen every week to receive study medication, check diary cards, record any unscheduled visits to a healthcare provider, and note the occurrence of any adverse events (AEs). CT scans were performed at baseline and at 12 and 24 months, and there were options for additional scans at 3 and 30 months (see online supplementary material for details). Post-bronchodilator lung function and health status were assessed at baseline and at $6,12,18,24$ and 30 months.

\section{CT densitometry end-points}

The progression rate of emphysema was determined by the change in the 15th percentile lung density (PD15) derived from the CT voxel distribution histogram of the whole lung. (For details of the CT scanner protocols and image analysis please see the online supplementary material.)

The reproducibility of level of inspiration during scan acquisition is recognised to influence lung density and reduces the reproducibility of CT lung densitometry. Consequently, different methods of adjustment for variation in inspiratory level between scans were explored: two methods were used to standardise density values for total lung volume (TLV), measured from CT, and the data were analysed using two different statistical approaches, resulting in four different analytical methods (fig. 1).

In Methods 1 and 3, PD15 was adjusted in individual patient scan series to a ratio of TLV divided by the individual patient's predicted total lung capacity (TLC) ("physiological adjustment"; see online supplementary material for further details) $[17,18]$. In Methods 2 and 4, adjustment was made by including $\log (\mathrm{TLV})$ as a covariate in the statistical model ("statistical adjustment"; see Statistical analysis section for further details) $[9,19]$.

\section{Other end-points}

Lung function (FEV1, diffusing capacity of the lung for carbon monoxide $(D \mathrm{~L}, \mathrm{CO})$ and transfer coefficient of the lung for carbon monoxide (KCO)) was assessed by post-bronchodilator spirometry and the single-breath method according to European guidelines [17]. The frequency of exacerbations was collected using patient diary cards and case-report forms, and exacerbations were defined according to the criteria described by RODRIGUEZ-RoISIN [20]. Moderate exacerbations were those requiring treatment with antibiotics and/or systemic corticosteroids. Exacerbations requiring emergency room treatment or hospitalisation were considered to be severe. Health status was assessed according to scores on the St George's Respiratory Questionnaire (SGRQ) [21].

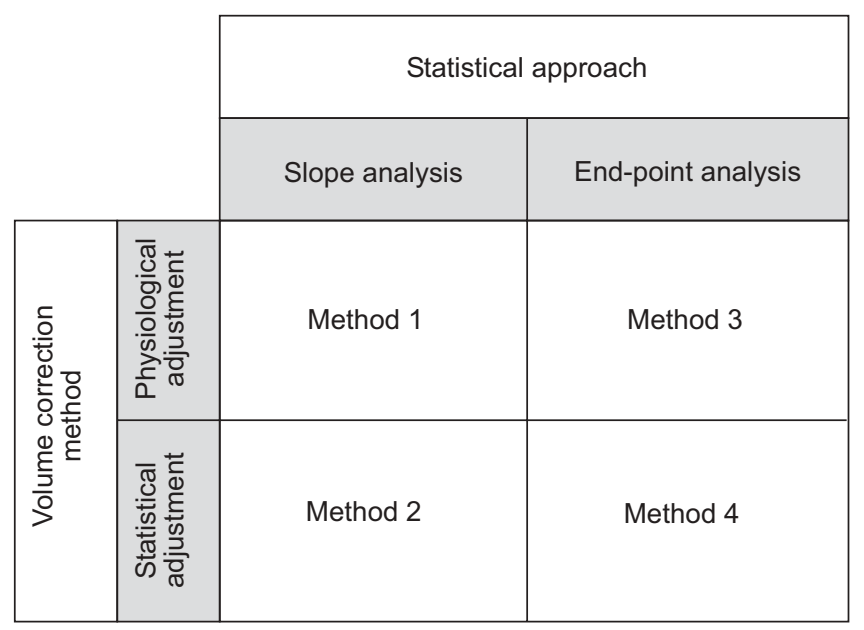

FIGURE 1. The four methods that were used for densitometric analysis 


\section{Statistical analysis}

Analysis of the study population included intent-to-treat (ITT) and modified intent-to-treat (mITT) populations. The ITT population included all randomised subjects. The mITT population comprised the ITT population excluding patients with fewer than two valid CT scans (baseline and 12 months or after). Prior to unblinding, a review panel assessed CT scan data to identify invalid scans due to technical issues (see online supplementary material). These values were excluded from further statistical analyses. All CT scan analyses were based on the mITT population, whereas analyses on other end-points used the ITT population.

In Methods 1 and 2 for the densitometric analysis (fig. 1), treatment differences (Prolastin ${ }_{\circledR}$ versus placebo) were tested by linear regression on time of PD15 measurement in a random coefficient regression model as follows. Method 1: TLCadjusted PD15 from CT scan as the dependent variable; treatment, centre and treatment by time interaction as the fixed effects; and intercept and time as the random effects. Method 2: PD15 from CT scan as the dependent variable; treatment, centre and treatment by time interaction as the fixed effects; logarithm of TLV as a time-dependent covariate; and intercept and time as the random effects. The estimated mean slope for each treatment group represented the rate of lung density change with respect to time. The tested treatment difference was the estimated difference in slope between the two groups, considered to be equivalent to the difference in the rates of emphysema progression.
In Methods 3 and 4, only the first and last available CT scans were used in an end-point analysis (main effect ANCOVA model), either with the physiological adjustment (Method 3) or by including $\log$ (TLV) as covariate in the model (Method 4). The effect variables and covariates were as follows. Method 3: change from baseline to the last CT scan measurement in TLCadjusted PD15 as the dependent variable; treatment and centre as fixed factors; and baseline measurement as covariate. Method 4: change from baseline to the last CT scan measurement in PD15 as the dependent variable; treatment and centre as fixed factors; and change in logarithm of CT-measured TLV and baseline measurement as covariates.

Before unblinding, Method 1 was identified as the primary end-point and Methods 2, 3 and 4 were identified as secondary CT outcomes. For the other efficacy variables, either the random coefficient model or the Cochran-Mantel-Haenszel test was employed. This was an exploratory study; for practical reasons we did not power the trial as a definitive study for proving a beneficial effect of augmentation therapy for any of the efficacy end-points. The statistical analyses were performed using PROC MIXED procedure in SAS version 9.13 (SAS Institute Inc., Cary, NC, USA).

\section{RESULTS}

Patient disposition and baseline characteristics

Patient disposition is summarised in figure 2. Of the 82 patients enrolled into the study from the three centres, 77 patients were randomised to Prolastin ${ }(n=38)$ or placebo

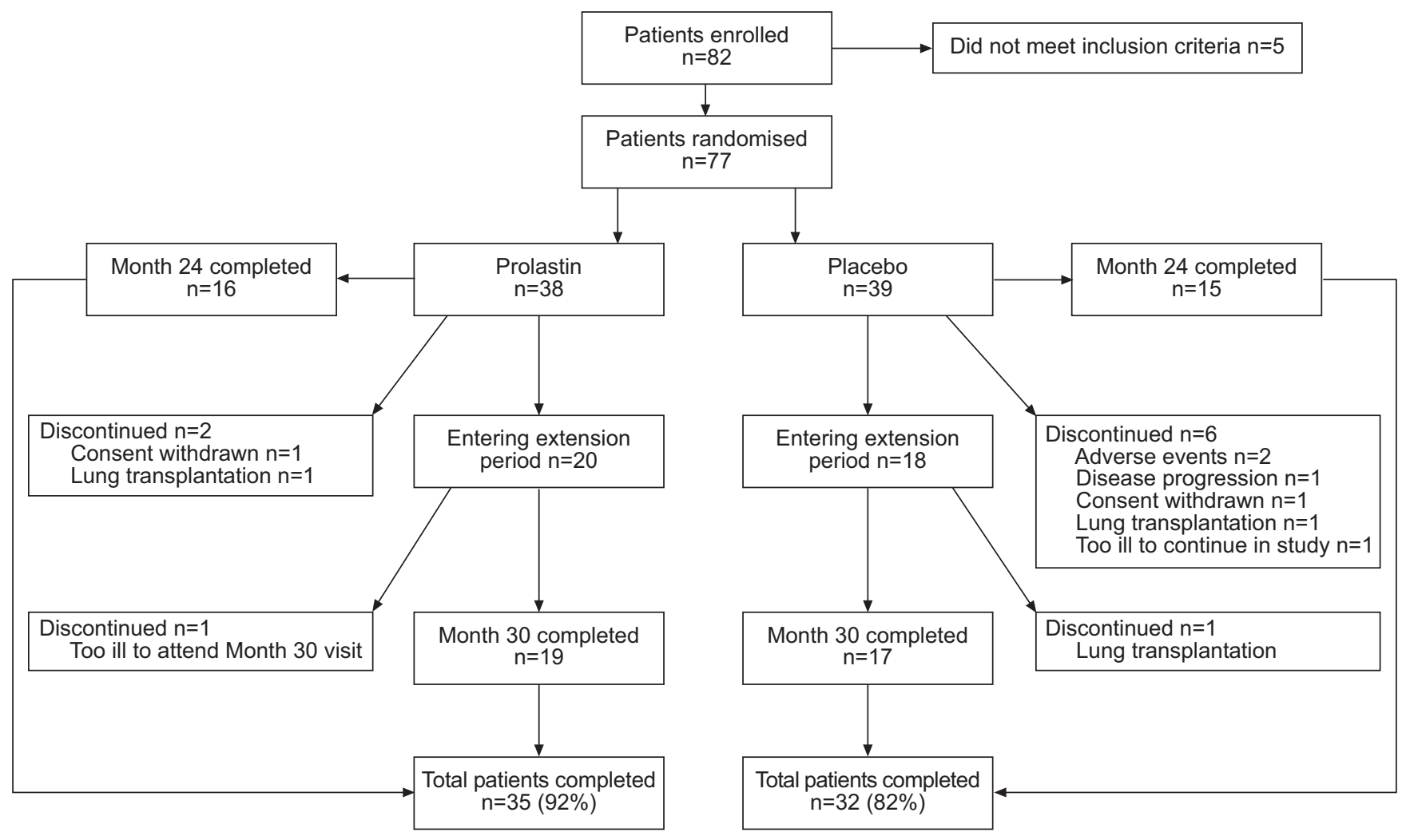

FIGURE 2. Flow of participants through each stage of the randomised trial. Planned study end (completion of the study) was Month 24 for those who did not enter into the extension period and Month 30 for those who entered into the extension period. Numbers are shown for the intent-to-treat population, which included all randomised subjects. 


\begin{tabular}{|c|c|c|c|}
\hline \multirow[t]{2}{*}{ TABLE 1} & \multirow[b]{2}{*}{ Prolastin ${ }_{\mathbb{B}}$} & \multirow[b]{2}{*}{ Placebo } & \multirow[b]{2}{*}{ p-value } \\
\hline & & & \\
\hline Subjects $\mathrm{n}$ & 38 & 39 & \\
\hline Age yrs & $54.7 \pm 8.4$ & $55.3 \pm 9.8$ & 0.749 \\
\hline Sex (males/females) $n$ & $25 / 13$ & $16 / 23$ & 0.021 \\
\hline $\begin{array}{l}\text { Smoking status } \\
\text { (never/ex-smokers) } n\end{array}$ & $4 / 34$ & $4 / 35$ & 0.949 \\
\hline Caucasian race $n$ & 38 & 39 & NA \\
\hline BMI $\mathbf{k g} \cdot \mathrm{m}^{-2}$ & $24.3 \pm 3.2$ & $24.3 \pm 3.5$ & 0.928 \\
\hline$\alpha_{1}$-AT levels $\mu \mathrm{M}$ & $4.6 \pm 1.6$ & $4.6 \pm 1.7$ & 0.898 \\
\hline $\begin{array}{c}\text { Genotype } \mathrm{ZZ} \text { and/or } \\
\text { phenotype } \mathrm{PiZ} n\end{array}$ & 38 & 39 & NA \\
\hline FEV 1 L & $1.44 \pm 0.60$ & $1.35 \pm 0.62$ & 0.542 \\
\hline FEV $1 \%$ pred & $46.3 \pm 19.6$ & $46.6 \pm 21.0$ & 0.873 \\
\hline$D \mathrm{~L}, \mathrm{CO} \mathrm{mmol} \cdot \mathrm{min}^{-1} \cdot \mathrm{kPa}^{-1}$ & $4.73 \pm 2.09$ & $4.72 \pm 1.70$ & 0.956 \\
\hline$D$ L,co \% pred & $50.7 \pm 19.5$ & $52.2 \pm 15.2$ & 0.721 \\
\hline $\mathrm{Kco} \mathrm{mmol} \cdot \mathrm{min}^{-1} \cdot \mathrm{kPa}^{-1} \cdot \mathrm{L}^{-1}$ & $0.82 \pm 0.32$ & $0.86 \pm 0.24$ & 0.565 \\
\hline Kco \% pred & $55.3 \pm 21.0$ & $56.5 \pm 14.8$ & 0.816 \\
\hline SGRQ Total score & $41.9 \pm 17.9$ & $46.1 \pm 17.2$ & 0.335 \\
\hline $\mathrm{PD}^{\prime} 5^{\#} \mathrm{~g} \cdot \mathrm{L}^{-1}$ & $47.98 \pm 19.07$ & $45.48 \pm 16.95$ & 0.288 \\
\hline TLC-adjusted PD15 ${ }^{\#} \mathrm{~g} \cdot \mathrm{L}^{-1}$ & $54.55 \pm 17.37$ & $53.90 \pm 15.97$ & 0.823 \\
\hline Lung weight ${ }^{\#} \mathrm{~g}$ & $956.40 \pm 140.64$ & $946.09 \pm 224.12$ & 0.750 \\
\hline Lung volume $e^{\#} \mathbf{L}$ & $7.46 \pm 1.60$ & $7.27 \pm 1.78$ & 0.557 \\
\hline
\end{tabular}

Data are presented as mean $\pm \mathrm{SD}$, unless otherwise stated. BMI: body mass index; $\alpha_{1}$-AT: $\alpha_{1}$-antitrypsin; PiZ: protease inhibitor type Z; FEV1: forced expiratory volume in $1 \mathrm{~s} ; \%$ pred: \% predicted; $D \mathrm{~L}, \mathrm{CO}$ : diffusing capacity of the lung for carbon monoxide; $K \mathrm{CO}$ : transfer coefficient of the lung for carbon monoxide; SGRQ: St George's Respiratory Questionnaire; PD15: 15th percentile lung density; TLC: total lung capacity; NA: not applicable. ${ }^{\#}$ : for the computed tomography densitometric analyses, the modified intent-to-treat population was used (Prolastin $n=36$; placebo $n=35$ ).

$(n=39)$, and 71 patients (Prolastin ${ }_{\mathbb{1}} \mathrm{n}=36$; placebo $\mathrm{n}=35$ ) were included in the mITT population. The number of patients in the ITT population who completed the study (either 24 or 30 months; fig. 2) was 67, and 10 patients (three in the Prolastin ${ }^{\circledR}$ group and seven in the placebo group) discontinued prematurely, resulting in a median of 127 weeks of exposure to Prolastin $_{\circledR}$ and 108 weeks to placebo. In terms of the mITT population, the study was completed by $34(94 \%)$ and $31(89 \%)$ patients in the Prolastin ${ }^{\circledR}$ and placebo groups, respectively.

Overall, demographics and disease severity for patients at baseline were well distributed between the groups (table 1). There were some sex differences between the treatment groups, with more males in the Prolastin ${ }$ treatment group and more females in the placebo group $(p=0.021)$. There were also sex differences between participating centres: in the UK there were more males enrolled into the study, and in Sweden there were more females. In Denmark, conversely, males and females were equally distributed. All patients fulfilled the physiological inclusion criteria, except for two patients with FEV1 baseline values slightly below 25\% predicted.

\section{CT densitometric progression}

A total of 15 scans were considered to be technically invalid, which resulted in six patients having fewer than two CT scans, and therefore these patients were excluded from the mITT population (for further details see the online supplementary material). CT densitometry demonstrated a significant decline in both the Prolastin ${ }^{\circledR}$ and placebo groups over the course of the study $(\mathrm{p}<0.001$ for both groups), consistent with emphysema progression.

The mean decline in whole lung density as measured by PD15 was determined using each of the four exploratory methods (tables 2 and 3). A difference in the decline of lung density

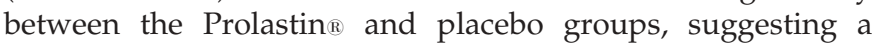

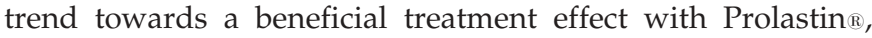
was seen consistently with all four analytical methods ( $p$ values for treatment difference ranged 0.049-0.084). Furthermore, the change in TLC-adjusted PD15 from baseline over the course of the study determined using Method 1 showed that the difference in lung density between the two treatment groups increased with time (fig. 3). Whereas there was a significant difference in the measured decline in lung density between the centres $(p<0.001)$, no significant interaction between centre and treatment was found. This indicated that the variation between the centres did not have an impact on the differences in the treatment effect observed between the two groups.

Mean values of lung volume as assessed by CT remained almost unchanged over the course of the study (see table 2 in the online supplementary material). However, there was wide variation in lung volume between scans in individual patients. A plot of change in lung density against change in lung volume from baseline is shown in figure 4 . A significant decrease $(p<0.001)$ in lung density was observed for both treatment groups when there was no change in lung volume (i.e. $\triangle \mathrm{TLV}=0$ ). This indicated that a proportion of the loss of lung density was not secondary to progressive hyperinflation but was consistent with an absolute loss of lung mass, which was greater in the placebo group. These data were further supported by the observation that mean values of CTmeasured lung weight decreased from baseline in both the Prolastin ${ }_{\circledR}$ and placebo groups during the study. Comparison of the slopes showed that there was a greater decrease in lung weight in the placebo group, although the treatment difference was not statistically significant (see table 3 in the online supplementary material).

PD15 was a more sensitive measure of emphysema progression than conventional physiological and health status indices (table 4). Of the different exploratory methods used to analyse PD15, Method 4 was the most sensitive.

\section{Other efficacy end-points}

Values for FEV1, DL,CO and KCO decreased slightly in both treatment groups during the study but, since these measures were less sensitive than $\mathrm{CT}$, no significant differences were found between the groups (see online supplementary material for details).

The mean $\pm S D$ annual exacerbation rates in the Prolastin ${ }^{\circledR}$ and placebo groups were similar $(2.55 \pm 2.14$ and $2.19 \pm 1.33$, respectively; $p=0.265)$, with a median frequency of 2.02 exacerbations $\mathrm{yr}^{-1}$ for both groups. A post hoc analysis, however, indicated that the Prolastin ${ }^{\circledR}$ group had proportionately fewer severe exacerbations, in relation to the total 


\begin{tabular}{|c|c|c|c|c|c|}
\hline \multirow{3}{*}{\multicolumn{2}{|c|}{ TABLE 2}} & capacity (TLC)-ac & and statistically ad & 15th percentile lun & ity (PD15) using slope \\
\hline & & \multicolumn{2}{|c|}{ Method 1 (Physiological) } & \multicolumn{2}{|c|}{ Method 2 (Statistical) ${ }^{+}$} \\
\hline & & Prolastin $\bar{B}$ & Placebo & Prolastin $_{\mathbb{B}}$ & Placebo \\
\hline Subjects $n$ & & 36 & 35 & 36 & 35 \\
\hline Month $24^{f}$ & & $-2.827 \pm 5.009$ & $-4.209 \pm 3.447$ & $-2.386 \pm 5.253$ & $-3.810 \pm 4.062$ \\
\hline Month $30^{\#}$ & & $-3.252 \pm 4.498$ & $-4.707 \pm 4.512$ & $-2.408 \pm 4.296$ & $-3.462 \pm 4.691$ \\
\hline $\begin{array}{l}\text { Random co } \\
\text { model me }\end{array}$ & $\begin{array}{l}\text { afficient regression } \\
\text { an slope }{ }^{-\varphi} \mathrm{g} \cdot \mathrm{L}^{-1}\end{array}$ & $-1.384(-2.023--0.745)$ & $-2.241(-2.905--1.577)$ & $-1.114(-1.618--0.611)$ & $-1.814(-2.339--1.289)$ \\
\hline $\begin{array}{l}\text { Estimated tr } \\
\text { mean slop }\end{array}$ & $\begin{array}{l}\text { eatment difference in } \\
\mathrm{g} \cdot \mathrm{L}^{-1}\end{array}$ & \multicolumn{2}{|c|}{$0.857(-0.065-1.778)$} & \multicolumn{2}{|c|}{$0.700(-0.028-1.427)$} \\
\hline
\end{tabular}

number of exacerbations with known severity, than the placebo group (13 (6.7\%) out of 194 versus 21 (13.5\%) out of 155 , respectively; $\mathrm{p}=0.013)$. This concurred with there being fewer subjects in the Prolastin ${ }_{\mathbb{B}}$ group with at least one hospitalisation due to an exacerbation during the course of the study (6 $(15.8 \%)$ out of 38 subjects in the Prolastin ${ }^{\circledR}$ group versus $11(28.2 \%)$ out of 39 in the placebo group; $\mathrm{p}=0.189)$.

Patients showed markedly impaired health-related quality of life at baseline on the SGRQ (table 1). At study end-point (the last post-baseline measurement for each subject), mean SGRQ 'Total' domain scores had increased (i.e. worsened) by 1.48 in the Prolastin ${ }^{\circledR}$ group and by 2.37 in the placebo group $(\mathrm{p}=0.695)$.

\section{Relationship between CT densitometry and other outcome measures}

We found a statistically significant relationship between the progression of CT densitometry and the rate of decline in FEV1 (fig. 5), but the correlation between loss of CT lung density and the rate of decline in $\mathrm{KCO}$ or health status indices was not statistically significant (data not shown).

\section{Safety}

All but one subject in each group experienced at least one AE. More than $90 \%$ of these AEs were classified as mild or moderate. There were 14 drug-related AEs in the Prolastin ${ }_{\mathbb{}}$ group and 35 in the placebo group, which were reported in $28.9 \%$ and $38.5 \%$ of subjects in each group, respectively. This

TABLE 3 Changes in total lung capacity (TLC)-adjusted and statistically adjusted 15th percentile lung density (PD15) using endpoint analysis ${ }^{\#}$

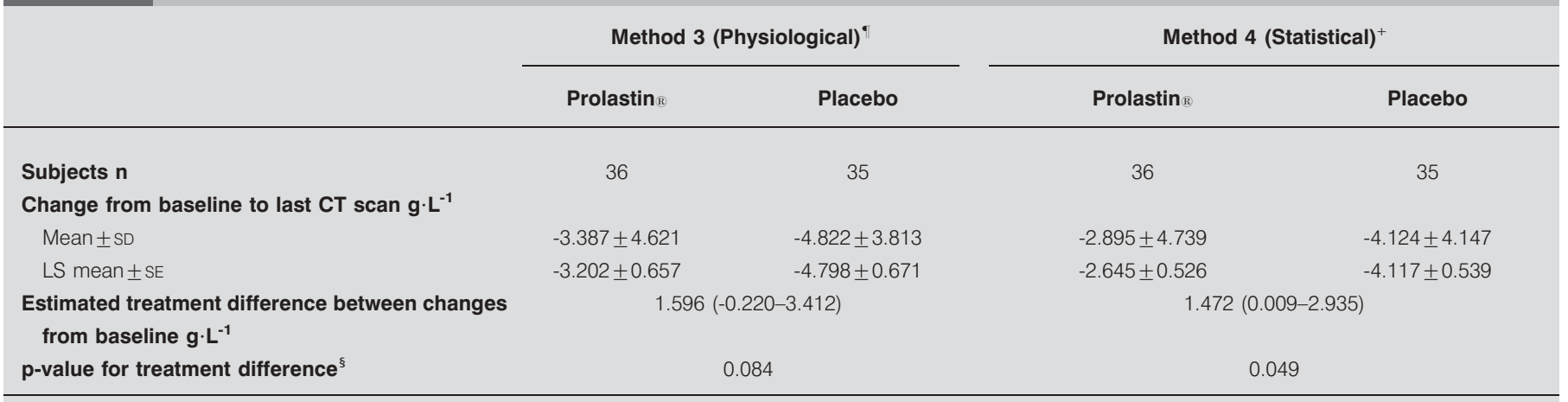

Data are presented as value (95\% confidence interval), unless otherwise stated. CT: computed tomography; LS: least squares. ${ }^{\#}$ : modified intent-to-treat population

$\because$ : change from baseline to the last CT scan measurement in TLC-adjusted PD15 as the dependent variable; treatment and centre as fixed factors; and baseline measurement as covariate. ${ }^{+}$: change from baseline to the last CT scan measurement in PD15 as the dependent variable; treatment and centre as fixed factors; and change in logarithm of CT-measured total lung volume and baseline measurement as covariates. ${ }^{\S}$ : Prolastin® treatment minus placebo. 


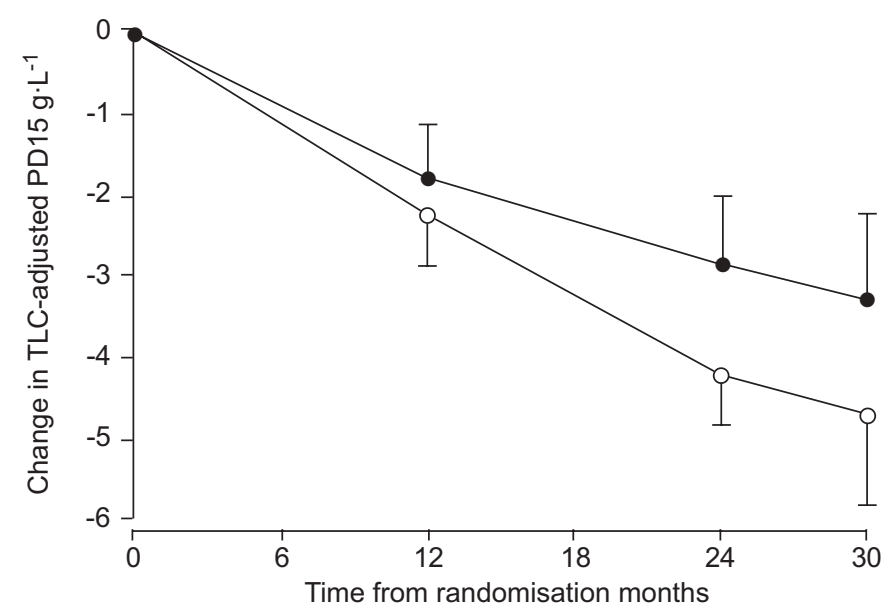

FIGURE 3. Change from baseline in total lung capacity (TLC)-adjusted 15th percentile lung density (PD15) over the course of the study using Method 1 for densitometric analysis on the modified intent-to-treat population. - Prolastin®; $O$ : placebo. The error bars indicate SEM. Estimated treatment difference in mean slopes (annual change in lung density) $0.857(p=0.068)$.

equates to an incidence of 0.004 and 0.009 drug-related AEs per infusion of Prolastin ${ }^{\circledR}$ and placebo, respectively. A total of 28 serious AEs (SAEs) occurred in $10(26.3 \%)$ subjects in the Prolastin $_{\mathbb{R}}$ group, and 40 SAEs were reported in $18(46.2 \%)$ subjects in the placebo group. A listing of the numbers of subjects with SAEs is shown in table 4 in the online supplementary material. One SAE was assessed as drugrelated in the Prolastin ${ }^{\circledR}$ group (psoriasis), and one in the placebo group (pulmonary embolism). Overall, two patients in the placebo group and none in the Prolastin ${ }^{\circ}$ group withdrew owing to AEs.

\section{DISCUSSION}

The current study was designed to prospectively explore the use of novel outcome measures as an alternative to traditional methods, such as FEV1, to assess the influence of $\alpha_{1}$-AT augmentation therapy on emphysema progression in $\alpha_{1}$-ATdeficient individuals. The data confirm the findings of previous studies [10, 22, 23] by demonstrating that CT densitometry is a more sensitive measure for the detection of emphysema progression than both physiological and health status indices. Consequently, it is predictable that the use of CT densitometry as the primary outcome measure in studies of emphysema-modifying therapy may be more likely to identify a treatment effect than traditional measures such as FEV1. The current study did identify a trend that was suggestive of a beneficial effect of augmentation therapy when assessed by CT densitometry and, furthermore, this trend was not evident when assessed by FEV1. Similar findings have also been shown in a previous randomised placebo-controlled clinical trial that was of comparable design [10]. It is of interest that these two studies have generated data suggestive of an equivalent treatment effect when assessed by CT densitometry, whereas the reduction in the rate of decline in FEV1 that had been demonstrated in previous observational studies [5-8] has not been reproduced. This latter finding, while disappointing, is both unsurprising and predictable, since CT has been repeatedly shown to be more sensitive than other measures

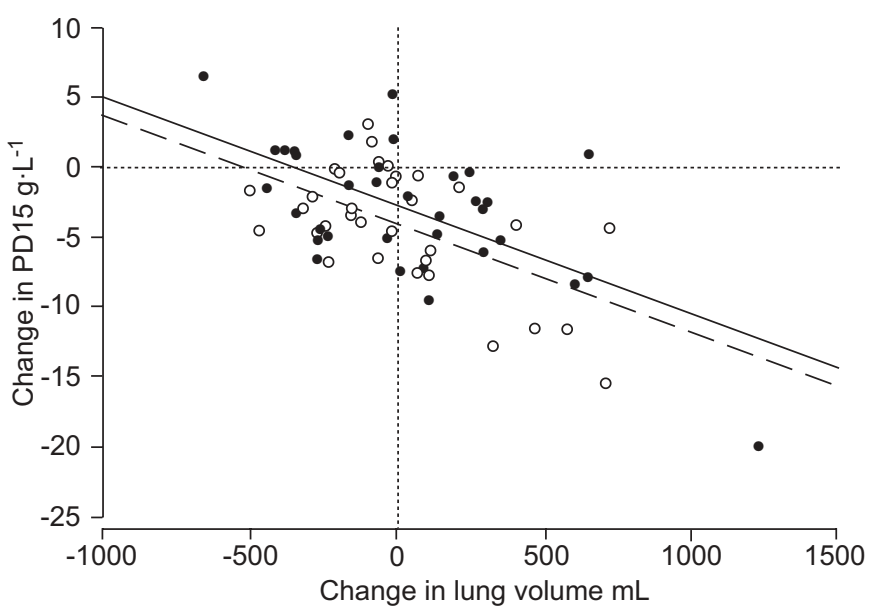

FIGURE 4. Plot and regression line of change from baseline to end-point in statistically adjusted 15th percentile lung density (PD15) versus lung volume, using the modified intent-to-treat population. - and ---: Prolastin®; $\bigcirc$ and --- : placebo

of emphysema progression [9, 22, 24], and power calculations would indicate that no treatment effect would be expected using FEV1 in such a small sample size [2, 3].

Notwithstanding the relative insensitivity of FEV1 to detect emphysema progression, the current data, however, also demonstrate that CT densitometric progression is related to the rate of decline in FEV1, as previously shown in an observational study [14]. Although there are differences in the scanning protocol, in the population demographics and in the use of augmentation therapy in half of the subjects in the current study, the regression equations derived from these two datasets are very similar, suggesting that a unit change in lung density may be equated with a recognisable effect in terms of FEV1. The correlation observed between densitometric and physiological declines may seem counter-intuitive when the treatment effect that is suggested by differences in the decline of densitometric indices is not evident in the decline in FEV1. However, this is not an inconsistent finding but rather a reflection of the lower sensitivity of FEV1 arising from poor reproducibility (as evidenced by the greater SE of this measure; table 4) and, although FEV1 is less sensitive than CT densitometry in monitoring emphysema progression, it does correlate with CT in cross-sectional studies [12, 14].

While these two confirmatory findings are of critical importance in further validating the methodology of CT densitometry, the primary purpose of the EXACTLE trial was to investigate alternative approaches to the analysis of CT densitometric data and to resolve several issues for which there remains a lack of consensus. We used PD15 as the CT end-point for the following reasons. First, the sensitivity of the voxel index method is threshold dependent, whereas the effect on sensitivity of using different percentiles is less evident with the percentile density method [9]. Consequently, the rate of decline in PD15 is more consistent across a wide spectrum of disease severity, whereas the progression of the relative area of emphysema below -950 HU (RA-950) increases with greater disease severity [14]. Secondly, PD15 has been gradually 


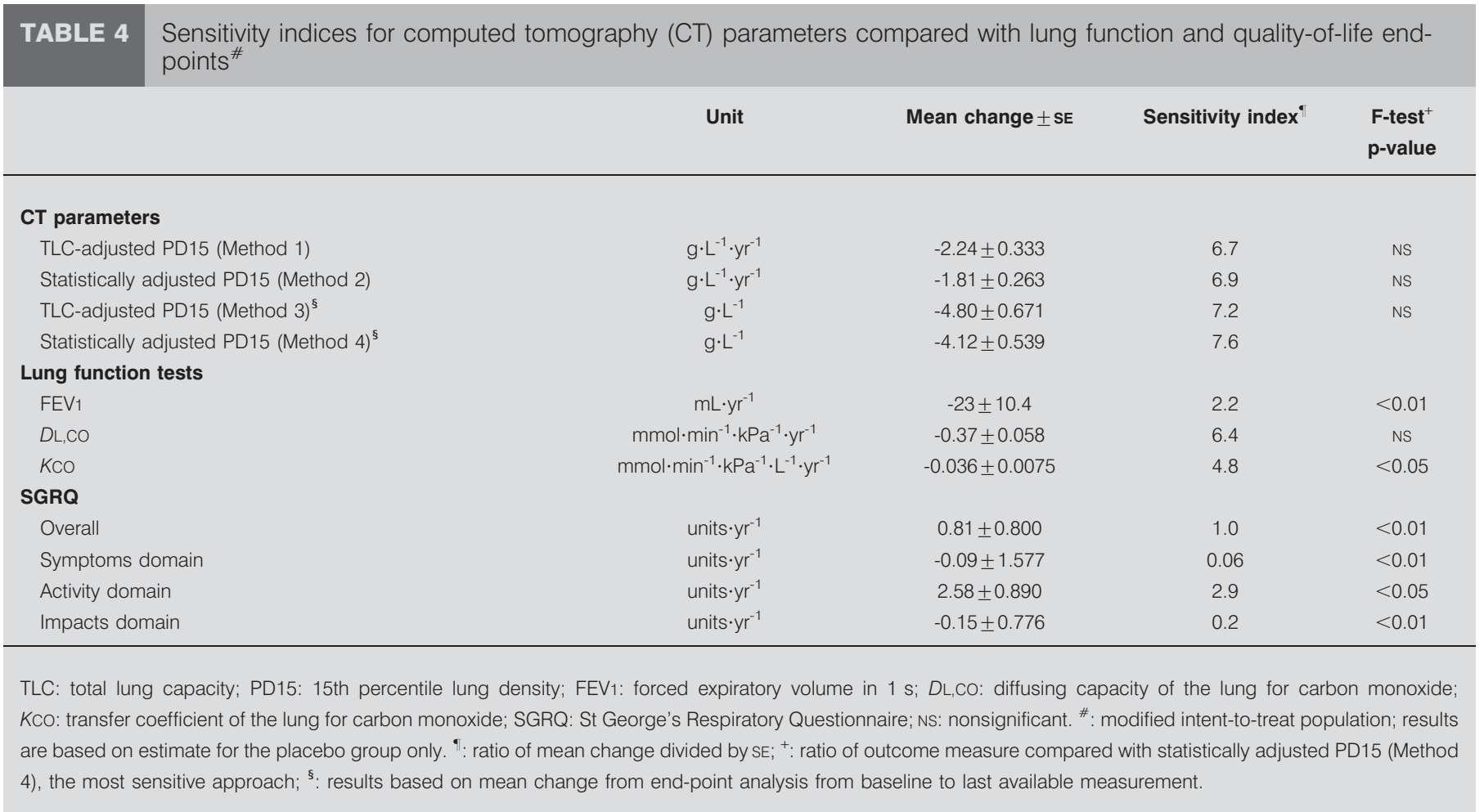

accepted as the most sensitive parameter for CT lung densitometry $[9,14,19]$. The workshop on quantitative CT scanning in longitudinal studies of emphysema recommended that the primary end-point should be a significant shift in the PD15 [25]. However, a broad range of data was collected in order to allow a flexible analytical approach that would be able to take account of interim developments in a rapidly changing field. We will address some of these approaches, including RA950 and regional analysis, in future studies.

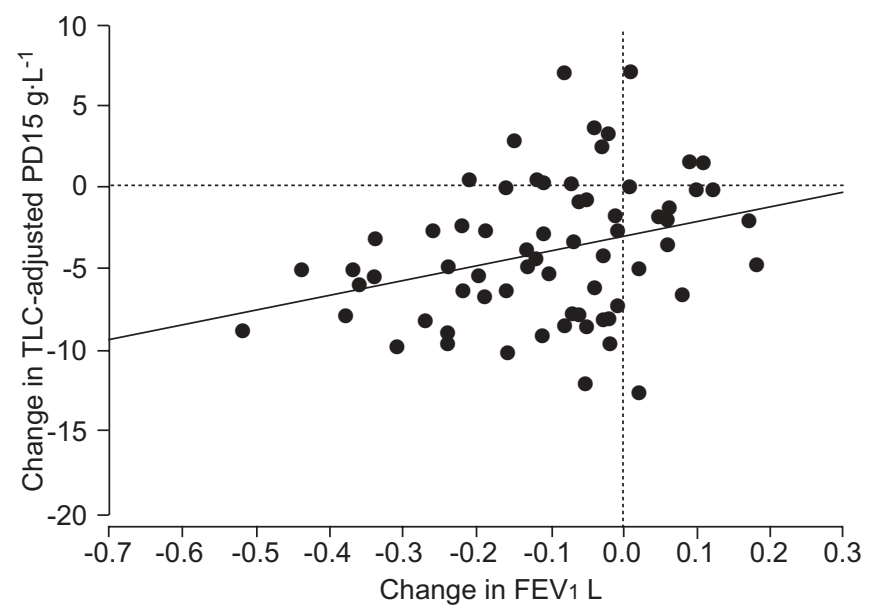

FIGURE 5. Correlation between the change in total lung capacity (TLC)adjusted 15th percentile lung density (PD15) versus change in forced expiratory volume in $1 \mathrm{~s}\left(\mathrm{FEV}_{1}\right)$, using the modified intent-to-treat population and both Prolastin $\mathbb{B}$ and placebo groups combined. Correlation coefficient $0.316 ; p=0.007$.
The principal clinical confounder in densitometric reproducibility is the level of inspiration during scan acquisition, and controlling or adjusting for this factor is the key to improving physiological variability. The current study addressed this issue by incorporating two methods for volume adjustment. The inverse relationship that exists between lung volume and lung density $[18,19]$ enables density values to be a priori adjusted by a physiological adjustment (Methods 1 and 3). As an alternative method, an a posteriori statistical adjustment was performed by the inclusion of the logarithmically transformed TLV as a covariate in the statistical model (Methods 2 and 4). The two statistical methods of analysis that were applied to these data were the mixed-effects, random coefficient model (Methods 1 and 2) and an end-point analysis (Methods 3 and 4). The former is a comprehensive statistical method that takes into account all measurements from baseline to end-point and allows for variation in the rate of progression of the disease between patients within the same treatment group. The latter, however, may be preferred in some situations (such as regulatory submission), to show the treatment effect between two groups in change from baseline to the end of the study. Due to the significant difference in the measured decline in lung density between the centres, all statistical analyses included the centre as a fixed effect. The significant centre effect seen in the EXACTLE study implies that in future studies the centre should be included in the statistical model.

Use of the current study methods of volume adjustment improved the reproducibility of CT densitometry, and statistically significant lung density loss was demonstrated in both treatment groups, consistent with emphysema progression. The decline in the group receiving $\alpha_{1}$-AT augmentation therapy was less than that in the placebo group, and this was consistently evident with all four methods ( $p$-values for 
treatment difference, Prolastin ${ }^{\circledR}$ versus placebo, ranged 0.049-0.084). When the method of volume correction used was similar to that of the previous randomised placebocontrolled clinical trial of $\alpha_{1}$-AT augmentation (Method 2) [10], a comparable treatment effect was observed. The similarity between the findings of the two studies when using a comparable analytical method would suggest not only consistency of treatment effect, but also robustness of CT densitometry as an outcome measure.

A linear relationship between the rate of change in lung density and the rate of change in lung volume, as identified in a recent observational study, was seen in both treatment arms; this enabled densitometric values to be adjusted for lung volume using statistical modelling, as described previously [19]. In addition, the intercepts for both treatment groups $(\Delta \mathrm{TLV}=0)$ were significantly different from zero $(\mathrm{p}<0.001)$, consistent with absolute loss of lung mass, also shown in the recent observational study [19]. However, there was a difference in the intercept between the active and placebo arms, which is consistent with a protective effect of augmentation therapy, as hypothesised in the observational study [19]. This overall observation is of fundamental importance in understanding the therapeutic effects of $\alpha_{1^{-}}$ AT augmentation therapy and would refute the potential dissenting argument that modification of densitometric progression might only reflect a reduction in lung compliance. Furthermore, it provides evidence to support the proteaseantiprotease hypothesis of proteolytic destruction without complete regeneration of tissue in the pathogenesis of emphysema [26].

With respect to the other outcome measures, no significant difference was observed between the two groups in the change in scores for the 'Total' domain of the SGRQ. Additionally, only insignificant differences were seen with regard to decline in FEV1, DL,CO or KCO. This last observation is not unexpected in view of the small size of the patient cohort in the present study. However, the CT densitometric progression identified in the present randomised placebo-controlled trial may be compared with the findings of previous observational studies that used lung function measures of assessment and that suggested clinical efficacy of augmentation therapy [5-8]. An approximation of the clinical relevance of the treatment effect in the present study may be made by reference to previously published studies [14, 24]. A treatment effect of $1.472 \mathrm{~g} \cdot \mathrm{L}^{-1}$, as demonstrated using Method 4, could equate approximately to a difference in FEV1 decline of $30 \mathrm{~mL} \cdot \mathrm{yr}^{-1}$ [14], which would be comparable with the treatment differences of 21 and $27 \mathrm{~mL} \cdot \mathrm{yr}^{-1}$ that were seen in individuals with moderate-tosevere lung function impairment in the previous observational studies $[5,7]$.

This is the first study to prospectively investigate the effect of augmentation therapy on exacerbations as a secondary endpoint. Exacerbations are a major cause of morbidity and mortality in general COPD [27] and are related to the decline in lung function, as measured by vital capacity and DL,CO [22], in $\alpha_{1}$-AT deficiency. These episodes are associated with greater neutrophilic inflammation and more free elastase activity in $\alpha_{1^{-}}$ AT deficiency compared with usual COPD [28], and retrospective data have suggested that exacerbations in $\alpha_{1}$-AT deficiency were less frequent once augmentation therapy was started [29]. The average annual exacerbation rate in the present study was similar in both the treated and placebo groups and was comparable with results for patients with $\alpha_{1^{-}}$ AT deficiency from the UK Registry who were not receiving augmentation therapy [30]. The number of patients included was too small to draw any conclusion on the treatment effect on the rate of exacerbations. In addition, it remains possible that there is a qualitative change in the episodes. HILL et al. [28] demonstrated a greater degree of inflammation in exacerbations in patients with $\alpha_{1}$-AT deficiency compared with those in patients with no $\alpha_{1}$-AT deficiency. It is therefore likely that the episodes in patients who received $\alpha_{1}$-AT augmentation therapy may be associated with less inflammation, although further studies will be needed to specifically address this issue. While the absolute number of exacerbations was no different in the current study, the severity as defined by healthcare utilisation was different, with fewer hospital admissions being required for subjects receiving $\alpha_{1}$-AT augmentation therapy. In the present study, augmentation therapy was generally shown to be well tolerated, with a favourable safety profile in accordance with findings from previous studies [6].

In conclusion, disease progression was demonstrated in both treatment groups using CT densitometry as a surrogate marker of emphysema. CT densitometry was more sensitive than other outcome measures in detecting disease progression and was also related to the decline in FEV1. Trends towards a deceleration of emphysema progression were observed for $\alpha_{1}$-AT augmentation therapy and were consistently seen using the different analytical approaches that were explored in this study. In future studies concerning the analysis of timedependent nonlinear treatment effects, Method 1 would be preferred, although it is less sensitive and thus requires more patients to detect treatment differences. In order to demonstrate the superiority of one treatment over the other, Method 4 appears to be the most sensitive and requires the smallest number of patients in a trial. Furthermore, the legal and ethical obligation to minimise exposure to ionising radiation in the course of clinical studies is also of paramount importance when considering the design of studies that utilise CT lung densitometry [31]. These results indicate that CT densitometry, performed as part of a multicentre study, is a technically feasible outcome measure in therapeutic trials of emphysemamodifying therapy.

\section{ACKNOWLEDGEMENTS}

Editorial assistance was provided by M. Kenig at PAREXEL (Worthing, UK) and was supported by Talecris Biotherapeutics, Inc.

\section{REFERENCES}

1 Snider GL, Lucey EC, Christensen TG, et al. Emphysema and bronchial secretory cell metaplasia induced in hamsters by human neutrophil products. Am Rev Respir Dis 1984; 129: 155-160.

2 Schluchter MD, Stoller JK, Barker AF, et al. Feasibility of a clinical trial of augmentation therapy for $\alpha_{1}$-antitrypsin deficiency. The Alpha-1-Antitrypsin Deficiency Registry Study Group. Am J Respir Crit Care Med 2000; 161: 796-801. 
3 Burrows B. A clinical trial of efficacy of antiproteolytic therapy: can it be done? Am Rev Respir Dis 1983; 127: S42-S43.

4 Wewers MD, Casolaro MA, Sellers SE, et al. Replacement therapy for $\alpha_{1}$-antitrypsin deficiency associated with emphysema. N Engl J Med 1987; 316: 1055-1062.

5 Seersholm N, Wencker M, Banik $N$, et al. Does $\alpha_{1^{-}}$ antitrypsin augmentation therapy slow the annual decline in FEV 1 in patients with severe hereditary $\alpha_{1}$-antitrypsin deficiency? Wissenschaftliche Arbeitsgemeinschaft zur Therapie von Lungenerkrankungen (WATL) $\alpha_{1}$-AT study group. Eur Respir J 1997; 10: 2260-2263.

6 Wencker M, Banik N, Buhl R, et al. Long-term treatment of $\alpha_{1}$-antitrypsin deficiency-related pulmonary emphysema with human $\alpha_{1}$-antitrypsin. Wissenschaftliche Arbeitsgemeinschaft zur Therapie von Lungenerkrankungen (WATL) $\alpha_{1}$-AT study group. Eur Respir J 1998; 11: 428-433.

7 Survival and FEV1 decline in individuals with severe deficiency of $\alpha_{1}$-antitrypsin. The Alpha-1-Antitrypsin Deficiency Registry Study Group. Am J Respir Crit Care Med 1998; 158: 49-59.

8 Wencker M, Fuhrmann B, Banik N, et al. Longitudinal follow-up of patients with $\alpha_{1}$-protease inhibitor deficiency before and during therapy with IV $\alpha_{1}$-protease inhibitor. Chest 2001; 119: 737-744.

9 Dirksen A, Friis M, Olesen KP, et al. Progress of emphysema in severe $\alpha_{1}$-antitrypsin deficiency as assessed by annual CT. Acta Radiol 1997; 38: 826-832.

10 Dirksen A, Dijkman JH, Madsen F, et al. A randomized clinical trial of $\alpha_{1}$-antitrypsin augmentation therapy. Am J Respir Crit Care Med 1999; 160: 1468-1472.

11 Spouge D, Mayo JR, Cardoso W, et al. Panacinar emphysema: CT and pathologic findings. J Comput Assist Tomogr 1993; 17: 710-713.

12 Gevenois PA, De Vuyst P, de Maertelaer V, et al. Comparison of computed density and microscopic morphometry in pulmonary emphysema. Am J Respir Crit Care Med 1996; 154: 187-192.

13 Dowson LJ, Newall C, Guest PJ, et al. Exercise capacity predicts health status in $\alpha_{1}$-antitrypsin deficiency. Am J Respir Crit Care Med 2001; 163: 936-941.

14 Parr DG, Stoel BC, Stolk J, et al. Validation of computed tomographic lung densitometry for monitoring emphysema in $\alpha_{1}$-antitrypsin deficiency. Thorax 2006; 61: 485-490.

15 Knudson RJ, Standen JR, Kaltenborn WT, et al. Expiratory computed tomography for assessment of suspected pulmonary emphysema. Chest 1991; 99: 1357-1366.

16 Dawkins PA, Dowson LJ, Guest PJ, et al. Predictors of mortality in $\alpha_{1}$-antitrypsin deficiency. Thorax 2003; 58: 1020-1026.

17 Quanjer PH, Tammeling GJ, Cotes JE, et al. Lung volumes and forced ventilatory flows. Report Working Party Standardization of Lung Function Tests, European
Community for Steel and Coal. Official Statement of the European Respiratory Society. Eur Respir J 1993; 6: Suppl. $16,5-40$.

18 Shaker SB, Dirksen A, Laursen LC, et al. Volume adjustment of lung density by computed tomography scans in patients with emphysema. Acta Radiol 2004; 45: 417-423.

19 Parr DG, Sevenoaks M, Deng C, et al. Detection of emphysema progression in $\alpha_{1}$-antitrypsin deficiency using CT densitometry; methodological advances. Respir Res 2008; 9: 21.

20 Rodriguez-Roisin R. Toward a consensus definition for COPD exacerbations. Chest 2000; 117: Suppl. 2, 398S-401S

21 Jones PW, Quirk FH, Baveystock CM, et al. A self-complete measure of health status for chronic airflow limitation. The St George's Respiratory Questionnaire. Am Rev Respir Dis 1992; 145: 1321-1327.

22 Dowson LJ, Guest PJ, Stockley RA. Longitudinal changes in physiological, radiological, and health status measurements in $\alpha_{1}$-antitrypsin deficiency and factors associated with decline. Am J Respir Crit Care Med 2001; 164: 1805-1809.

23 Bakker ME, Stolk J, Putter $\mathrm{H}$, et al. Variability in densitometric assessment of pulmonary emphysema with computed tomography. Invest Radiol 2005; 40: 777-783.

24 Stolk J, Ng WH, Bakker ME, et al. Correlation between annual change in health status and computer tomography derived lung density in subjects with $\alpha_{1}$-antitrypsin deficiency. Thorax 2003; 58: 1027-1030.

25 Newell JD Jr, Hogg JC, Snider GL. Report of a workshop: quantitative computed tomography scanning in longitudinal studies of emphysema. Eur Respir J 2004; 23: 769-775.

26 Stockley RA. Neutrophils and protease/antiprotease imbalance. Am J Respir Crit Care Med 1999; 160: S49-S52.

27 Seemungal TA, Donaldson GC, Paul EA, et al. Effect of exacerbation on quality of life in patients with chronic obstructive pulmonary disease. Am J Respir Crit Care Med 1998; 157: 1418-1422.

28 Hill AT, Campbell EJ, Bayley DL, et al. Evidence for excessive bronchial inflammation during an acute exacerbation of chronic obstructive pulmonary disease in patients with $\alpha_{1}$-antitrypsin deficiency (PiZ). Am J Respir Crit Care Med 1999; 160: 1968-1975.

29 Lieberman J. Augmentation therapy reduces frequency of lung infections in antitrypsin deficiency: a new hypothesis with supporting data. Chest 2000; 118: 1480-1485.

30 Needham M, Stockley RA. Exacerbations in $\alpha_{1}$-antitrypsin deficiency. Eur Respir J 2005; 25: 992-1000.

31 Laying down basic safety standards for the protection of the health of workers and the general public against the dangers arising from ionizing radiation. Council Directive 96/29/EURATOM. Brussels, The Council of the European Union, 1996. 